\title{
Kyasanur Forest Disease: A Tropical Disease of the Southwest India
}

\author{
Mythreyi R, Geethanjali B, Kanthesh M Basalingappa, \\ Gopenath TS, Parthiban R and S Raviraja \\ ${ }^{1}$ Division of Molecular Biology, Faculty of Life Sciences, JSS AHER, SS Nagara, Mysuru, India \\ ${ }^{2}$ Department of Biotechnology \& Bioinformatics, Faculty of Life Sciences, JSS AHER, SS Nagara, Mysuru \\ ${ }^{3}$ Department of Clinical Laboratory Services \& Translational Research, Malabar Cancer Centre, Thalassery \\ ${ }^{4}$ University of Garden City, Khartoum, Sudan and PI, Royal Research Foundation, Karnataka, India
}

\section{ABSTRACT}

In 1957, the unexpected epizootic outbreak of an unknown virus in the vicinity Kyasanur forest of Shimoga district's Sagara taluk, led to the fatality of a set of mammals, including humans. The disease is called Kyasanur Forest Disease [KFD] and the causal organism is Kyasanur Forest Disease Virus [KFDV], a flavivirus. KFD is classified as a tick-borne viral hemorrhagic fever, reporting 400 to 500 cases per year with a mortality rate of 2-10\%. The virus exhibits genetic and clinical similarity with documented Alkhurma virus in Saudi Arabia, Russian encephalitis, Osmak hemorrhagic fever, documented in China as Nanjianyin virus. During year 2010, it open casted around the Egypt-Sudan region and It was found that this fever was also carried by arachnoid of Ornithodorids and other genera, and which parasitise sheep and camels. Despite the similarity with other viruses, KFDV remains endemic to Southwestern parts of India, especially to the Karnataka region of Malandu. however, having observed at recent cases reported in states such as Goa, Kerala, Maharashtra and Tamil Nadu, the health and science departments have been keen to conduct further research on KFDV. The proposed research work is an attempt to spread the evidence-based knowledge and awareness of the disease and related research in the field.

KEY WORDS: KYASANUR FOREST DISEASE (KFD), KYASANUR FOREST DISEASE VIRUS (KFDV), VIRAL HEMORRHAGIC FEVER.

\section{INTRODUCTION}

Human beings have always been affected by external forces and mainly by microorganisms, in the terms of health issues and to the extreme cases of death. These minuscule giants have a stronghold on the governance of the quality and the life span of most life on this planet. The facts about the human life, bacteria, virus, fungi, and protozoans play a vital role in the diseases and infections causation. Passim the history of mankind, viruses have

Biosc Biotech Res Comm P-ISSN: 0974-6455 E-ISSN: 2321-4007

\section{crossef}

Identifiers and Pagination

Year: 2021 Vol: 14 No (5) Special Issue

Pages: 145-153

This is an open access article under Creative

Commons License Attribn 4.0 Intl (CC-BY).

DOI: $h t t p: / / d x$.doi.org/10.21786/bbrc/14.5/28 been an almost undefeatable antagonist, whose regional and global outbreaks have claimed uncountable lives and the most recent of infectious virus being the coronavirus [Covid-19]. Currently with the advent of modern technology and scientific knowledge, it is known that there are probable about 219 species of viruses that cause diseases and show pathogenicity in human beings. The unpredictable nature, the high rate of mutation and adaptability, the cross-species contamination serve to the better nature of the viruses and these factors play an important role in causing setbacks in developing vaccines or cures to the respective diseases.

The Kyasanur Forest Disease (KFD) is a tropical disease that originated in India's Western Ghats. The disease is caused by an arbovirus called KFDV that belongs to the family Flaviviridae and spreads via ticks. This disease is also called Monkey Fever due to it being the
Article Information

Received: $19^{\text {th }}$ Jan 2021

Accepted after revision: $26^{\text {th }}$ Mar 2021 
first epizootic outbreak found among Kyasanur Forest monkeys in the year 1957. Prior to the advent of the viral disease in the monkeys, there found to be no evidence of any viral disease with the said set of symptoms in the human population but after the infection spread amongst the monkeys, the virus crossed the species border and affected the human population in the villages surrounding the forests exponentially. KFD is described as a viral hemorrhagic fever that also exhibits some neurological features. The virus spreads out in the targeted population through the bite of the infected Ixodid tick of the genera Haemaphysalis.

Approximately 400-500 cases with a fatality rate of 2-10\% have been recorded annually since the outbreak. The Haemaphysalisspinigera tick is the virus reservoir that infects certain primates in the Malenadu region. The Shimoga District, Tirthahalli, Hosanagara and Sagara taluks were considered the disease's most affected regions. Monkeys, rodents and shrews are the common hosts for KFDV. Human beings serve as ultimate or dead-end hosts. The symptoms of KFD include high fever, headaches, myalgia; severe cases show bleeding from nasal cavity, throat and gastrointestinal tract as well. While the virus shows significant fatality rates there is no global spread of the virus for reasons unknown. Semnopithecus entellus and Macaca radiata wild monkeys are more susceptible to KFDV infection, and the infection spreads by contact with these dead infected monkeys as well as other dead infected primates. The virus has now been recorded in other South Indian states like Tamil Nadu, Goa, Maharashtra and many other states of India like Gujarat, Rajasthan, West Bengal and in Andaman- Nicobar Islands.

History: The first outbreak of Kyasanur forest disease commonly termed "the monkey fever" was seen in Kyasanur Forest in Shimoga district, India dated 1957. Numerous sick and dead monkeys, more likely termed Nonhuman primates (NHPs) were found dead in the region . It was considered a febrile disease, a human epidemic in the same year at the primary health care center, Ulvi of Sagara taluk, Shimoga district. Many historical studies say the virus epidemic started in early 1956 . Serological studies initially indicated that the novel virus is linked to the arbovirus complex of Russian spring-summer encephalitis (RSSE), currently referred to as the Tickborne encephalitis (TBE) sero-complex of Flaviviruses, in effect associated with neurological disorders. Clinical descriptions of KFD and Omsk hemorrhagic fever (OHF) show many similarities but not with neurological abnormalities in terms of hemorrhage.

A 1995 viral outbreak in Saudi Arabia's Jeddah province, named Alkhurma Hemorrhagic Fever Virus (AHFV) had similar symptoms of KFDV. Although several studies suggest that both KFDV and AHFV were first found in Africa and migrated to other parts of the world via migratory birds to India, Saudi Arabia, Sudan-Egypt border and China; diverged about 700 years ago, thus remaining geographically separate. Although the virus has been considered as endemic to the south-western part of the country, in a specific district of Karnataka, several cases have recently been recorded in other districts such as Uttara Kannada and Chamarajanagar, as well as in other neighboring states such as Kerala, Tamil Nadu, Goa and Maharashtra. Following the initial identification of the disease, there have been several fatal cases recorded in both humans and monkeys annually.

Causes: KFD is an arboviral disease, spreading through vectors of the arthropod. The causative agent is the KFDV which belongs to the Flaviviridae family. The RNA virus KFDV measures between $40-60 \mathrm{~nm}$ in diameter. The KFDV genome consists of 10,774 single-stranded, positivesense RNS nucleotides, encoding a single polyprotein of 3416 residue length which is post-translationally cleaved into three structural (C, prM / M, and E) and seven nonstructural (NS1, NS2a, NS2b, NS3, NS4a, NS4b, and NS5) proteins. The viral genome exhibits considerable similarity of more than 90\% AHFV and OHFS.

The virus transmission is primarily through the forest's contaminated tick bite. Two major tick families are found in this temperate region; Argasidae is the soft tick family and Ixodidae is the hard tick family. They each contain about 100 and 700 varieties. Tick transmitting the KFDV comes under the hard tick type that belongs to the genera Hemaphysalis, spiningera genus; altogether, the KFDV has been isolated from 16 different tick genus. The tick has a life span of 3 blood meals. The larvae hatch out of the egg and feed for several hours to days on the first host, fall off and mold into the nymphal stage. The nymph begins to search a second host. Usually, the first and second hosts belong to the same species but may sometimes differ.

For several days, the nymph feeds on the second host leaves, it and molds into an adult tick. The adult ticks now find a host for the third meal and feed on blood. During this phase, the adult tick's mate on the host, the female takes more blood after the mating and drops off to the ground and lays a gelatinous mass of eggs in a sheltered area containing several thousand eggs. The eggs hatch and follow the same four-stage life cycle and 3 meals in blood. The H.spinigera tick is used as a KFDV reservoir [Fig 1].

Figure 1: The ecological cycle of the Kyasanur Forest Disease virus is depicted in this diagram.

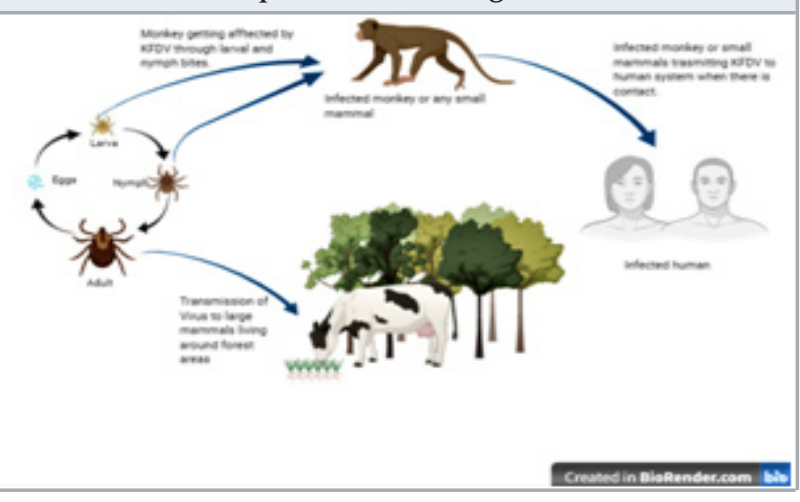


Ticks that are hard to kill the virus's reservoir and vector are both Haemaphysalis spinagera. Humans may contract the disease directly from infected ticks or indirectly from infected monkeys and small mammals. Larger animals may become infected, but they play a minor role in disease transmission to humans. It is widely seen that various wildlife play the role of host to many disease-causing organisms and the statistics that few of these disease-causing microorganisms can cause undesired effects in humansare medium to high. The wild Semnopithecus entellus monkeys and Macaca radiata are more prone to the virus infection. In these monkeys the epizootic leads to fatality. For humans, any direct interaction with an infected monkey or its dead body brings the disease. The bite of the specifically infected tick also triggers the infection of every primate of humans. But there is no record of KFDV transmission from humans to humans till date.

The risk factor recorded in the Western Ghats region is currently high. The risk of infection is seen in villages that have forest areas where mainly agriculture is practiced. The local people and forest officers are also at risk because of more land and animal contact. These animals live in the surrounding village areas and are at risk of infection with KFDV. It is observed that women are the most susceptive to the disease whereas the children are the least to be affected by this virus.

Clinical Features of the Disease: KFD is categorized by the International Classification of Diseases-10 (ICD-10), 2017, into group A98.2, which comes under other viral hemorrhagic fevers. KFDV shall have an incubation time of 3 to 8 days. This is also a biphasic condition where about 20 percent of the individuals affected show the onset of the disease in the 2 nd phase and 80 percent of them display the signs only in the first phase. Since the disease was characterized by biphasic anatomy, the first step (3-4 days) involves a sudden onset of high fever of around $400 \mathrm{C}$ or $1040 \mathrm{~F}$ and chills. The primary symptoms are general body pain in the neck, upper as well as lower back areas and headache. Gastrointestinal symptoms such as pain in the belly, vomiting, diarrhea, and dehydration are accompanied symptoms. It also detects conjunctival inflammation of both the sclera and the palpebra. Lymphadenopathy and hepato-splenomegaly are also documented in some cases but are non-specific features.

Throughout this process, the hemorrhagic manifestations are identified after the onset of the above-mentioned primary symptoms, starting with oral mucosal inflammation and non-tender maculopapular eruptions on both soft and hard palate, showing no allergic symptoms. Nearly every case includes ocular manifestations such as conjunctival congestion. In nearly 63 percent of cases, extreme discharge is involved, in 13 percent of cases, Retina and Vitreous humor are involved. Hematemesis is reported in 8 percent of cases, epistaxis in 2 percent, bleeding in rectum in 2 percent. Patients usually pass step one within 14 days. Patients can suffer from muscle twitching, coarse tremors, paraesthesia and generalized trembling due to weakness in the recovery time and will not be able to perform any physical activities.

Phase 2 symptoms are classified as anomalies in neurology. Only among 20\% of cases, it is published. This process includes fever with prevalent neurological symptoms such as drowsiness, intermittent disorientation, confusion, occasional seizures and loss of consciousness. The clinical symptoms found are Kernig symptoms and irregular ankle reflexes. No mention is made of meningitis and encephalitis. While the risk of fatality is around 2-10\%, many patients return to normal mental and physical orientation unless there are serious hemorrhagic conditions. Fatality is more likely in the elderly and patients with comorbid illnesses, liver and drinking disorders.

Pathology: Despite there being a huge number of cases affected by the virus being observed annually, the exact pathogenic mechanism or the host-to-parasite interaction is not known. There are many theories and speculations as to this infection being something more than just a simple acute febrile illness. The proposed mechanism of the disease is the onset of multiple pathological pathways that lead to multiorgan illness [Fig 2].

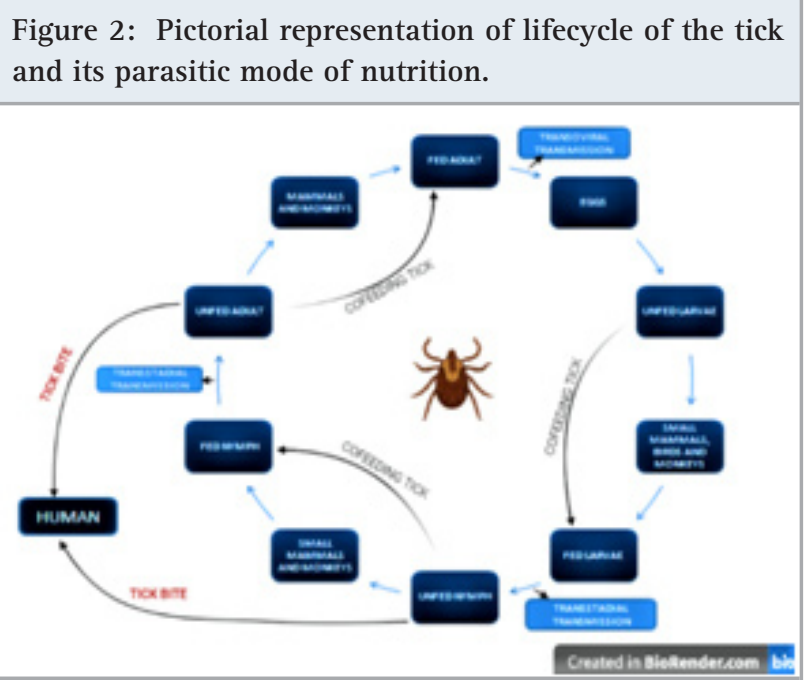

The study of the cellular mechanism of the infected system shows the virus upon entering the system nullifies the immune system of the host by targeting the IFN response and thereby inhibiting the JAK-STAT pathway. This mechanism is said to help the viral particles to replicate indefinitely and without any hindrance from the host immune system. Several theories state that the viral replication machinery aids in the viral particle to easily bind to the cell surface and thereby easily manipulating the cellular- cytoplasmic pathways that further weaken the host cell. Therefore, it is understood that the disease begins at the expense of partial destruction of the host's immune system. Following the uninterrupted replication of viral particles, there is an unnatural level of viral titer value and this is understood to be one of the many factors contributing to the expression of viral hemorrhagic fever along with several other disease symptoms. 
This proposed theory of mechanism is observed in many viral infections that follow a similar set of symptoms like Ebola found in Africa, Lassa viral diseases. In all these diseases, the infected APCs behave like a carrier of the viral particles and this helps the spread of the particles to regions like the spleen, liver and many organs that are rather immunologically well protected. This mechanism explains the pantropic nature of the virus [Fig 3].

Figure 3: The proposed mechanism of the establishment of the disease in monkeys and humans.

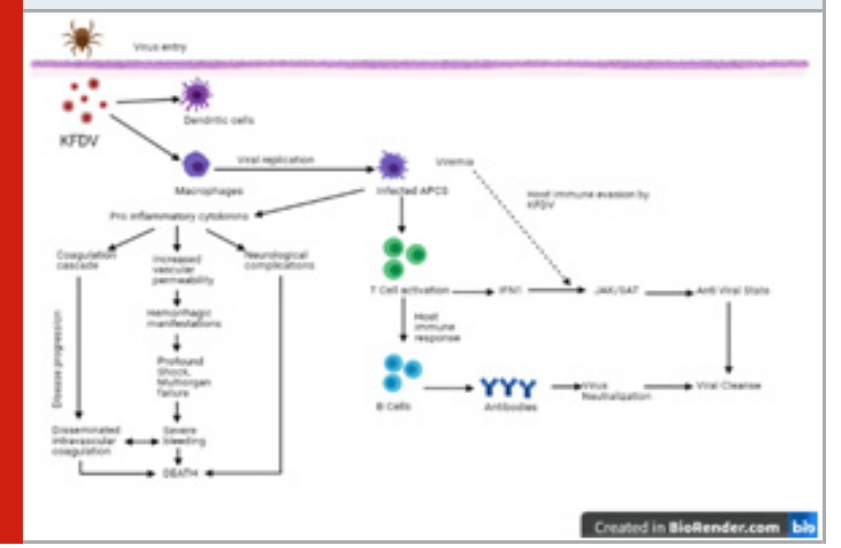

As mentioned above gross and microscopic examination of fatal cases of KFD have found evidence of a mostly non-specific disease process, displaying prominence and excessive liver and spleen macrophages and lymphocytes. There was significant mild parenchymal degeneration in the liver and kidneys as well as signs of spleen erythrophagocytosis. In most cases, the typical reporting was focused on necrosis in the liver and tubular damage in the kidney. Often the cervical and axillary lymph nodes get enlarged. Some cases have been recorded with hemorrhagic pneumonia. In certain cases, neurological symptoms such as aseptic meningitis are found along with cerebral edema is identified or partial infiltration of inflammatory cells. No report concluding encephalitis, meningitis and CSF abnormality as KFD pathological evidence. The interaction between the viral particles and their host is not well investigated and this is due to incomplete knowledge that we have in terms of the genomic makeup of the virus as well as the pathogenic and defense mechanism of the virus. To further investigate the pathogenicity and the mechanism of the establishment of the disease, several animal studies have been carried out in mice and bonnet macaques.

Genome and Phylogeny: KFDV is a Flavivirus which belongs to the pathogen community Biosafety level-4 (BSL-4). The viral genome consists of the positive-sense, single-stranded RNA measuring around $11 \mathrm{~kb}$ in length. This RNA encodes a single 3416 amino acid Polypeptide. These 3416 aa polypeptides are post-translationally cleaved into 10 proteins, including 3 structural proteins and 7 non-structural proteins as mentioned earlier. Capsid protein $\mathrm{C}$ which binds to the cell membrane and collects the viral RNA into a nucleocapsid forms the core of a mature virus particle that plays an important role in virus budding. The protein can induce genome penetration into the host cytoplasm following hemifusion induced by the surface proteins during virus entry. The protein can migrate to the cell nucleus where host functions are modulated and by interfering with host Dicer, it inhibits RNA silencing. The structural protein E proteins play a significant role in the infection of all the flaviviruses.

It binds to host receptors of the cell surface and mediates fusion between viral and cell membranes. In the endoplasmic reticulum the envelope protein is synthesized in the form of a heterodimer with protein prM. They play a role in virion budding in the ER, and the newly developed immature particle is covered by 60 spikes composed of a heterodimer between precursor prM and protein envelope E. The protein E forms dimers on the surface of the matured virions when the physiological $\mathrm{pH}$ is available; while the immature particles are covered by the protein heterodimers PrM-E. PrM serves as a chaperone for protein E during the assembly of the intracellular virion by masking and inactivating the protein E fusion peptide. The PrM covering immature particles avoid irreversible inactivation, as the virus is transported through the acidic compartments of the transGolgi network, thus preventing disastrous activation of the viral fusion. Pr peptide prevents premature fusion activity of envelope proteins in trans-Golgi by binding to envelope protein $\mathrm{E}$ at pH6.0, which is dissociated from $\mathrm{E}$ dimers after virion release in extracellular space. Small protein envelope M plays a role in budding viruses. Exerts cytotoxic effects by activating an apoptotic mitochondrial pathway via M ectodomain and displays viroporin activity [Fig 4].

Figure 4: Flavivirus organization, and structure. Picture courtesy: Theodore C. Pierson and Michael S. Diamond, 2020.

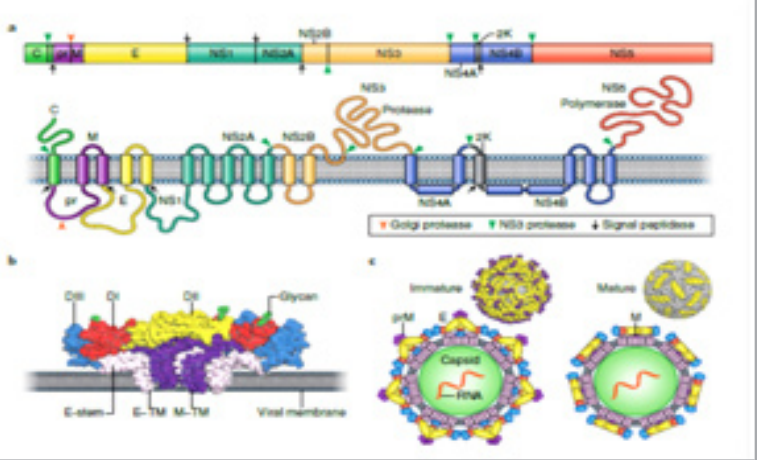

Flavivirus encodes a single open reading frame which is translated into a polyprotein at the ER and then cleaved by viral and host cell proteases. The structural component E proteins contribute to the virulence of the virus and are characterized by elongated three-domain structures connected by a stem and two antiparallel transmembrane domains to the viral membrane. The E protein domains are shown in red, yellow, and blue, respectively (DI - III). The M protein, which also has two transmembrane domains attached to the viral membrane, is shown in 
purple. C, the distinct arrangement of E proteins is shown on the immature (left) and mature (right) forms of the virion [Fig 5].

Figure 5: Homology model structure of the ectodomain (aa 1-394) of KFDV E Protein. Arrow indicates the position of fusogenic motif.

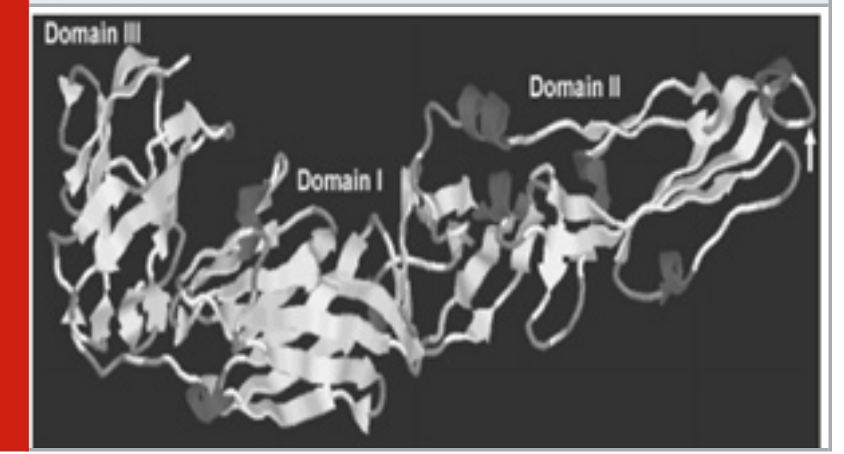

The non-structural NS1 protein (also observed in TBE) has immune evasive functions. NS1 protein induces oxidative stress and activates defense against antioxidants. NS1 protein includes the immune evasion, pathogenesis and replication of viruses. When the polyprotein has been cleared, three destinations are targeted: the process of viral replication, the membrane of the plasma and the extracellular compartment. It is necessary for viral replication needed to shape the complex replication and to recruit other non-structural proteins to the membrane structures derived from the ER. This is excreted as a hexameric lipo-particle that plays a part against the immune response from the host.

It binds to macrophages and dendritic cells of the host and prevents signal transduction coming from Toll-like 3 (TLR3) receptors. NS2a protein is a component of the complex for viral RNA replication that functions in the assembly of virions and antagonizes the immune response of the host. The N terminal of NS3 protein has a serine phosphate domain, requiring for its activity the membrane-bound NS2B protein cofactor and an ATPdriven helicase and RNA triphosphatase at its C-terminal end. NS4A Controls NS3 helicase activity on the ATPase. NS4A allows for energy conservation by NS3 helicase during unwinding.

NS4B induces the development of ER-derived membrane vesicles where viral replication occurs and inhibits interferon-induced STAT1 phosphorylation and nuclear translocation by blocking the IFN-alpha / beta pathway. Translocation of STAT2 is inhibited at the nucleus after treatment with IFN-alpha. Peptide $2 \mathrm{k}$ acts as an NS4B signal peptide and is necessary for the latter's interferon antagonism operation. NS5 protein replicates the viral (+) and (-) RNA genomes and performs cytoplasm-capping of genomes. NS5 is responsible for the methylation of viral RNA cap at N-7 guanine and at 2'-0 ribose positions. This also is responsible for preventing the establishment of cellular antiviral state by blocking the signaling pathway of interferon-alpha / beta (IFN-alpha / beta), in addition to its function in RNA genome replication. Inhibits phosphorylation of the host TYK2 and STAT2 and thus prevents activation of the JAK-STAT signaling pathway. NS5 is the enzyme replicator with methyltransferase and RNA polymerase activity [Table 1].

Based on the 48 whole genome sequences, the phylogenetic analysis identified a large community with good bootstrap support, consisting of the recent 2006 to 2017 strains. Although distinct, the older KFDV stresses from 1957 to 1972 did not form a homogeneous cluster. The overall nucleotide divergence across all KFDV strains based on the full genome was 2.24 percent, whereas it was 2.76 percent between the two groups. The average diversity of amino acids between all KFDV strains based on the complete genome was 0.75 percent, while 0.86 percent was between the two classes. The highest nucleotide difference between the two groups was observed in the capsid gene (3.27\%), followed by the gene E (3.22\%) and the gene NS2 (2.91\%).

\begin{tabular}{|c|c|c|c|c|c|}
\hline Gene & Primer & Genome location & Primer sequence & Product bp & Type \\
\hline \multirow[t]{4}{*}{ PreM-env } & KFD-EF2 & $459-478$ & TGGTGTTCTCGCGACAGTT & 780 & Genotyping \\
\hline & KFD-ER2 & $1258-1238$ & TCTGTCACTCTGGTCTCGCTT & 780 & Genotyping \\
\hline & KFD-EF3+ & $606-628$ & TCATTCGAGTGTGTGTCACCATT & 780 & Genotyping \\
\hline & KFD-ER1+ & $701-678$ & TTCCGTATTCCAGTGACACTCGCT & 780 & Genotyping \\
\hline \multirow[t]{4}{*}{ NS5 } & KFD-F3 & $9422-9441$ & GGCTGAGTCATGGACATCAT & 642 & Genotyping \\
\hline & KFD-R4 & $11046-11063$ & TCCACTCGTGTGGATCCT & 642 & Genotyping \\
\hline & KFD-F4+ & $9660-9680$ & TGAGACCTTCTGACGACCCCT & 642 & Genotyping \\
\hline & KFD-R3+ & $9801-9819$ & TCCTTATCGTCAACTCAT & 642 & Genotyping \\
\hline
\end{tabular}

In capsid protein (3.17\%), followed by prM (1.66 \%), and NS2 (1.4\%), the highest percentage of amino acid variation between the two classes was noted. The amino acid substitutions that delineated the two classes were C: N56S; prM: F130L and NS1: S271N, NS3: G221R. A comparison of the source species-wise divergence in the different genes showed that the highest nucleotide divergence $(2.39 \%)$ between human and tick species was observed, followed by that between human and monkey $(2.23 \%)$ and monkey and tick $(2.06 \%)$ respectively. The 
maximum nucleotide variations in the capsid gene were reported in all three species followed by the gene $\mathrm{E}$ and the gene NS2, respectively. Consideration of the expanded data collection of 76 E-gene sequences showed that the relative variance of nucleotides and amino acids was $2.61 \%$ and $0.33 \%$.

The above evolution tree shows the full genome phylogeny of the AHFV and KFDV and is based on the Bayesian coalescent analysis. The analysis shows those eons before the current period, both the virus had a common ancestor which then separated to evolve into the current KFDV and AHFV strains. It can be concluded that even though these two viruses show genetic similarity, these two viruses are not responsible for each other's evolutionary existence. This analysis tree shows us that the evolutionary roots of such viruses are deeper than our superficial understanding and need more research and understanding. The specific color-coordinated strains denote the effect the virus has ona certain host; the different strains show different host specificity. The green-colored strains show human host specificity, the blue color strains show tick pool specificity, and the orange strains show non-human primate host specificity.

Viral Replication: The first step of virus replication begins when the virus binds to the cell receptor that has asparagine-linked sugars with several cell-specific intercellular adhesion molecules and is taken up into the cell by endocytosis mediated by the receptor. The attachment is mediated on the host cell via viral $\mathrm{E}$ protein and entry receptors. The entry receptor for TBFVs has not been identified, but attachment to heparan sulfate and glycosaminoglycan is predicted to play a role during binding and entry, which are present in abundance on many cell types of both vertebrates and ticks. Subsequently reaching the cell, the virus is transported to endosomes where the acidic condition of these vesicles contributes to the reorganization and conformation of the E protein, resulting in the fusion of the viral and endosomal membrane and the release of the viral capsid into the cytoplasm.

Once inside the cell the viral genome codes for single open reading frames of mRNA that are flanked with highly untranslated regions [UTRs] that play various important functions in the maintenance of the viral replication and weakening the host immune system. Once the open frame enters the cytoplasm, multiple viral polyproteins are produced and these then target the endoplasmic reticulum organelle which favors the safe transport of the new viral particles. This step of the viral particles invading the ER is found to be an important step in the further synthesis of the components of the viral coat like NS1, NS2A, NS2, NS4A, and NS5 There are various changes in the biochemical metabolic nature of the host cell aided by the virus to create a favorable climate to produce the flavivirus replication complexes. These changes include the increase in the level of cholesterol and fatty acids, manipulation of the host enzymatic systems.
The viral protein's transmembrane domain is recognized as a signal peptide and recruits the vRNA / ribosomes / nascent polypeptide complex into the ER membrane where it is translocated co-translationally into the ER membrane. The nascent polypeptide is then processed into structural and non-structural (NS) viral proteins using the cellular and viral proteases. Several viral proteins, such as NS2B, NS4A, and NS4B, incorporate and modify the ER membrane to create a vesicular membrane structure, with a narrow pore connecting the vesicle's interior to the cytoplasm. These vesicles are the site where replication complexes (RC) and RNA replication can be produced. Following RNA replication, on the cytoplasmic side of the ER membrane, genomic RNA is proposed to exit the vesicle through the vesicular pore and is packaged into the NC by $\mathrm{C}$ protein. This acquires the lipid envelope along with $\mathrm{E}$ and prM proteins, which are aligned with the ER membrane, during the budding phase of NC from the cytoplasm into the ER.

The mechanism ensuring the effective integration of NC with the E and prM protein into the ER membrane is poorly understood. The subsequent budding into the ER lumen, the immature virions are transported to the extracellular medium in a COPI and COPII-dependent manner through the cellular secretory pathways. The immature virion has E and prM heterodimers that completely cover the lipid bilayer to form a spiky proteinaceous coat. The E protein is glycosylated during conveyance through the Golgi. The Golgi's acidic condition causes conformational changes in the proteins $\mathrm{E}$ and prM, which reveals a cleavage site on prM. PrM cleavage by the cellular protease furoin contributes to the development of a mature virion. Exocytosis then releases mature virions which completes the viral life cycle [Fig 6].

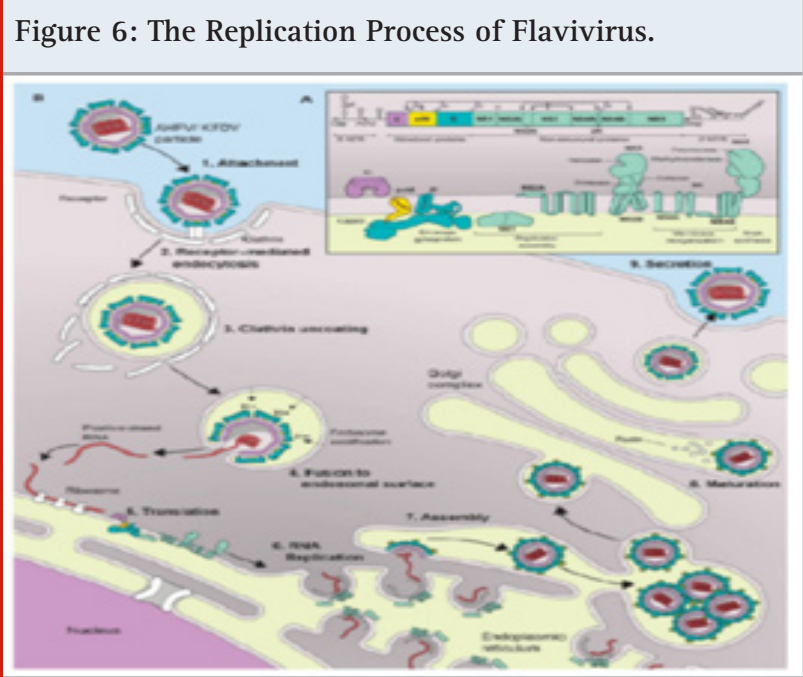

According to a study carried out in the Sindhudurg district of Maharashtra, India, on 72 individuals infected with KFD for 17 months by collecting their serum samples, KFD viral positivity was observed from the 1st to the 18th day after the start (POD). Positivity of anti-KFD virus (KFDV) antibodies IgM 4 to 122 POD and anti-KFDV IgG antibodies 5 to 474 PODs were 
observed. The study showed KFD viral RNA to 18 PODs, IgM antibodies to 122 PODs, and IgG to the last sample collected. An algorithm for exact laboratory diagnosis of KFDV infection was recommended based on our study. A sample collected between 1 and 3 POD may be tested using KFDV real-time reverse transcriptase-polymerase chain reaction (RT-PCR) tests; between 4 and 24 POD, the combination of real-time RT-PCR and anti-KFDV IgM enzyme-linked immunosorbent assay (ELISA) tests may be used; between POD 25 and 132, anti-KFDV IgM and IgG ELISA are recommended.

Diagnosis: KFD's early diagnosis is presently not a feasible feat, and this is attributed to the surface level understanding that we have about the virus and the tick mediated disease. The alleged case's blood serum was injected into the mice and kept under observation. The alleged cases are first seen for the presence of indicators like the presence of the infestation of infected vectors or infected monkeys, the history of the incidence of virus, and favorable climate for their replication. In the suspected case, the pathology and fatality display were used to confirm KFDV infection. Now, molecular methods such as PCR, RT-PCR are used to diagnose the disease in the early stages, since the technology has been developed. Serological monitoring is conducted in later stages using the Enzyme-Linked Immunosorbent Assay (ELISA).

The method of diagnosis is by using various biochemical and enzymatic assays like antibody binding ELISA, IFN $\alpha$ - ELISpot, virus neutralization or bactericidal immunoassay, biochemical -cellular methods that target certain antigen or derives proteins. Simple detection of pathogens from the blood sample or through tissue culture can also be used for the diagnosis. For any diagnosis to be useful, the reports must be backed with appropriate clinical assays that are sensitive and specific that are usually approved by WHO and FDA. That being said, there are only a few tests for emerging infectious diseases like KFD that have been titled with the Emergency Use Authorization [EUA].

There are various organizations and coalitions like the WHO, FDA, the Coalition for Epidemic Preparedness Innovations $\{\mathrm{CEPI}$ that work hard in establishing speedy response mechanisms, backup plans, and on-site diagnostic tools. The dire necessity of such mechanisms is seen in situations like the recent and ongoing pandemic COVID-19, where the lack of immediate action and the inadequate reaction has put the entire world on halt and the loss of lives is simply not to be accepted in the good grace of any consciousness.

Vaccination: Kyasanur Forest Disease is an animal to human infecting disease which is caused by a flavivirus called the Kyasanur Forest Disease Virus. Due to the incomplete information, we have on the virus and thereby its mechanism of pathology, we do not have a well-defined treatment course. The onset of 2015 saw the large-scale public vaccination plan of the people residing near hot zones of infection like Maharashtra, Karnataka, Goa, and others to reduce the disease from causing a fatality. In India, the vaccination initiation is a very important player in the control of the disease and the course of vaccination is carried out by vaccinating the people in two doses with less than 1-year time gap. The two-course dose system is employed due to the short period of immunity provided by one dose on children and adults. For practical purposes, annual booster doses are advised for about 5 years of period to ensure maximum protection .

The emerging and emergent nature of this virus makes the synthesis and mass production of a vaccine acts a challenge. Along with this challenge, the cost and commercialization of vaccines drive a stake against the balance to the clinical and health of the public issue of the disease. These challenges may be tackled by developing plans and understanding more about the socio-economic scenario of the targeted area and by following novel and innovative technologies like mobile laboratories. Understanding the rate at which the virus and the mode of contamination and spread is the key participant in deciding the fate of the vaccine production. The production and distribution of vaccines are handled through the Institute of Animal Health and Veterinary Biologicals in Bangalore, Karnataka. The vaccine was produced by using formalin-inactivated tissue culture of the organism that was taken from the previous infected human or primates. This mode of the vaccine was synthesized after many failed versions of the mouse brain and chick embryo based KFDV that were formalin inactivated. The reason for these vaccines to be scrapped was due to their lack of effectiveness or their lack in instigating a strong immune response.

The general role of vaccines is to function as a booster of the immune system and to provide a safe method of the system to recognize and become customized to the pathogen and thereby reducing the fatality factor of the disease. The introduction of vaccines against KFDV into the host causes the immune system to produce antibodies against the inactivated pathogen and in turn produce memory cells to the cell receptor of the pathogen. This is important in the effectiveness of the vaccine as these memory cells are the cells that produce active antibodies when the real pathogenic virus enters the body and henceforth produce a full-fledged protective response. As aforementioned, some studies have shown that once infected with the viral particles, there is a cellmediated cytoplasmic cascade mechanism of antibody production.

The initiation of IFN 1 production by the infected cells further pushes the production of antiviral chemicals. Both these mechanisms allow the biological system in attaining adaptive immunity against the virus. On the one-week post-infection mark, the production of antibodies aiding in complement fixation and blood coagulation is seen to increase. The onset of the second week is characterized by the production of antibodies that nullify the viral particles and the virus- antibody titer reaches the peak by the third-week timestamp. All these are achieved by the immune system along with antibodies 
neutralizing the E glycoprotein that is presented in the viral coat of the flavivirus which weakens and prevents the virus from attaching to the cell surface and stopping any further infection of healthy cells. The first diagnosis needs two series of dosages. This complicated vaccination schedule restricts vaccine absorption such that the priming sequence and first booster were provided by just one-third of subjects. The vaccine efficacy for fully vaccinated individuals was only 62 percent compared to 95 percent as originally reported, with major vaccine deficiency occurring the year after immunization.

\section{CONCLUSION}

Infections and diseases have always been ever-present either seasonal or annual in their pattern of presentation. With the advent of modern medicine and technology, humans are on an ever-changing path in finding cures and vaccines against such diseases. Studying nature and taking inspiration from nature herself to overcome problems has always been a long-time mechanics by which humans have devised and derived cure for diseases; although there are many cases where we do not succeed in establishing an upper hand over the disease and there are many case scenarios where researchers and scientists are still in the initial path of making vaccines.

The time that modern human has spent on Earth is relatively shorter than the time that certain microorganisms have existed. Thus, it can be easily concluded that we do not have all the information and understanding on these microorganisms. The fact that the rate of useful mutation and circumstantial evolution is faster and more visible in microorganisms and thereby with the advent of antimicrobial 'antibiotic' and 'vaccines' have pressured the microorganisms to evolve into a more virulent and dangerous strain that has more fatality percentage. The modern age of technology-aided with the help of science brought about more ways in which we could discover and analyze different species around us gave us the perception that there is a rise in the rate of virulent microorganisms while the truth is they were around from time immemorial and we were simply slow in discovering the required vaccine or ignored.

Viruses are one of the most confusing microorganisms that human beings have faced and that is credited to both their physical makeup that is simply made of an external protein coat and internal genetic material and their mystique nature of cellular interaction with the host. As previously mentioned, there are about 219 virus species that make humans their host and the first one of such viruses to be discovered was the yellow fever virus in the year 1901, and that began the era of viruses. Though viral diseases are fewer than bacterial, fungal, or parasitic diseases that affect mankind, the effect at which viral diseases affect human beings are simply incomparable to its contenders. This can be seen in the case of the latest and on-going global pandemic COVID-19.

One such recently discovered strain of the human infecting virus is the Kyasanur Forest Disease Virus.
KFD is an emerging viral disease transmitted by ticks and infected monkeys in India. The recent onset of the disease in other parts of India, such as Maharashtra, Goa, Tamil Nadu, and Kerala, has made it clear that the virus is spreading to other parts of India other than Karnataka, where the disease was first detected. This is considered a viral hemorrhagic fever with some or more characteristics of neurological disorders. The disease has a 2-10\% fatality rate and causes it. The virus shows genomic similarities but stands differently from fewer other viruses. Today, the vaccine's output has been drastically reduced from 95\% to 62\%, which demonstrates the need for further work in the field and the production of vaccines.

Ethical Approval and Consent to participate: Not applicable. The article does not include animal or human experiments.

Consent for publication: Neither the article nor portions of it have been previously published elsewhere. The manuscript is not under consideration for publication in another journal, and will not be submitted elsewhere until the journal Bioscience Biotechnology Research Communications, editorial process is completed. All authors consent to the publication of the manuscript in BBRC, should the article be accepted by the Editor-inchief upon completion of the refereeing process.

Availability of data and materials: All data generated or analyzed during this study are included in this published article.

Competing interests: The authors have no competing interest directly relevant to the content of this article.

Funding: Funding information is not applicable. No funding was received

Authors Contribution: Mythreyi R (MR) and Kanthesh M Basalingappa (KMB) conceptualized the study. Mythreyi R (MR) Geethanajali B (GB) Gopenath TS (TSG), drafted the Manuscript. Parthiban R (PR), S Raviraja (RS) and Kanthesh M Basalingappa (KMB) helped with the Manuscript and Discussion.

\section{ACKNOWLEDGEMENTS}

The authors would like to acknowledge the Management of JSS Academy of Higher Education \& Research, Mysuru, Karnataka, for supporting the basic research ideas and also for the resources provided.

\section{REFERENCES}

Annet Oliveira, Kalaiselvi Selvaraj, Jaya Prasad Tripathy, et al. Kyasanur Forest Disease Vaccination coverage and its perceived barriers in Goa, India- A mixedmethods operational research. PLOS ONE. 2019; 14 (2): e0226141.

Ashok Munivenkatappa1, Rima Rakesh Sahay2, Pragya D Yadav2, Rajalakshmi Vishwanathan3, Devendra T Mourya, Clinicaltt epidemiological significance of 
Kyasanur forest disease; Indian J Med Res. 2018 Aug; 148(2): 145-150.

Bhatia B, Feldmann H, Marzi A. Kyasanur Forest Disease and Alkhurma Hemorrhagic Fever Virus-Two Neglected Zoonotic Pathogens. Microorganisms. 2020 Sep 12;8(9):1406.

Christine C Roberts. Emerging Infectious disease laboratory and diagnostic preparedness to accelerate vaccine development. Hum VaccinImmunother. 2019; 15 (10): 2258-2263

Devendra T Mourya*, Pragya D. Yadav, Rajeev Mehla, Pradeep V. Barde, Prasanna N. Yergolkar, Sandeep R.P. Kumar, Jyotsna P. Thakare, Akhilesh P Mishra. Diagnosis of Kyasanur forest disease by nested RT-PCR, real-time RT-PCR and IgM capture ELISA;Journal of Virological Methods 186 (2012) 49- 54.

Dodd K.A., Bird B.H., Khristova M.L., Albarino C.G., Carroll S.A., Comer J.A., Erickson B.R., Rollin P.E., Nichol S.T. Ancient ancestry of KFDV and AHFV revealed by complete genome analyses of viruses isolated from ticks and mammalian hosts. PLoSNegl. Trop. Dis. 2011;5: e1352.

Jana Sirmarova, Jiri Salat, Martin Palus, Vaclav Hönig,et al.Kyasanur Forest disease virus infection activates human vascular endothelial cells and monocytederived dendritic cells; Emerg Microbes Infect. 2018; tandfonline; 7: 175.

Joel N. Maslow (2019) Challenges and solutions in the development of vaccines against emerging and neglected infectious diseases, Human Vaccines EtImmunotherapeutics, 15:10, 2230-2234, DOI: 10.1080/21645515.2019.1661209.

Joel N. Maslow. Challenges and solutions in the development of vaccines against emerging and neglected infectious diseases. Hum Vaccin IImmunother.2019;15(10):2230-2234.

Kapoor A, Slikas E, Simmonds P, Chieochansin T, Naeem A, Shaukat S, Alam MM, Sharif S, Angez M, Zaidi S, Delwart E. A newly identified bocavirus species in human stool. J Infect Dis. 2009; 199(2):196-200.

Lappalainen M, Niesters HGM. Introduction to Virus Diagnosis and Treatment. Reference Module in Life Sciences. 2020; B978-0-12-814515-9.00148-X. Lindqvist R, Upadhyay A, Överby AK. Tick-Borne Flaviviruses and the Type I Interferon Response. Viruses. 2018;10(7):340.

Mackenzie JS, Jeggo M. Reservoirs and vectors of emerging viruses. CurrOpinVirol. 2013 Apr;3(2):170-9.

Michael R. Holbrook. Kyasanur Forest Disease. Antiviral Rep. 2012; 96 (3): 353-362.
Pierson, T.C., Diamond, M.S. The continued threat of emerging flaviviruses. Nat Microbiol. 2020; 5, 796-812.

Pond SLK, Frost SDW.Datamonkey: rapid detection of selective pressure on individual sites of codon alignments. Bioinformatics. 2005; 21: 2531-2533.

Pragya D. Yadav, Savita Patil, Santoshkumar M. Jadhav, Dimpal A. Nyayanit, Vimal Kumar, Shilpi Jain, Jagadish Sampath, Devendra T. Mourya\&t Sarah S. Cherian; Phylogeography of Kyasanur Forest Disease virus in India (1957-2017) reveals evolution and spread in the Western Ghats region; Scientific Reports (2020) 10:1966.

Pragya D. Yadav, Yogesh K. Gurav, et al. Kinetics of viral RNA, immunoglobulin-M \& G antibodies in Kyasanur forest disease; Indian J Med Res. 2019 Aug; 150(2): 186-193.

PrinyabrataPattnaik. Kyasanur forest disease: an epidemiological view in India.Rev. Med. Virol. 2006; 16: 151-165.

Rajaiah P. Kyasanur Forest Disease in India: innovative options for intervention. Hum VaccinImmunother. 2019;15(10):2243-2248.

Rajeev Mehla, Sandeep R.P.Kumar, Pragya Yadav, et al.,. Recent Ancestry of Kyasanur Forest Disease Virus.2009; 15(9): 1431: 1437.

Shah SZ, Jabbar B, Ahmed N, et al. Epidemiology, Pathogenesis, and Control of a Tick-Borne DiseaseKyasanur Forest Disease: Current Status and Future Directions. Front Cell Infect Microbiol. 2018; 8:149.

SN Sharma1, Rina Kumawat2, Sujeet Kumar Singh3; Kyasanur Forest Disease: Vector Surveillance and its Control;Journal of Communicable Diseases Volume 51, Issue 2 - 2019; 38-44.

Tove Hoffman, Mats Lindeborg, Christos Barboutis, et al. Alkhurma Hemorrhagic Fever Virus RNA in HyalommarufipesTicks Infesting Migratory Birds, Europe, and Asia Minor; Emerging Infectious Diseases. www.cdc.gov/eid. 2018; 24(5).

Woolhouse M, Scott F, Hudson Z, Howey R, ChaseTopping M. Human viruses: discovery and emergence. Philos Trans R Soc Lond B Biol Sci. 2012;367(1604):28642871.

Work T. Russian spring-summer encephalitis virus in India. Kyasanur Forest disease. Prog. Med. Virol. 1958; 1:248-279.

Zaki A.M. Isolation of a flavivirus related to the tickborne encephalitis complex from human cases in Saudi Arabia. Trans. R. Soc. Trop. Med. Hyg. 1997; 91:179-181. 